\title{
Bound states of interacting helium atoms
}

\author{
Stefan V. Mashkevich ${ }^{\mathrm{a}}$ and Stanislav I. Vilchynskyy ${ }^{\mathrm{b}}$

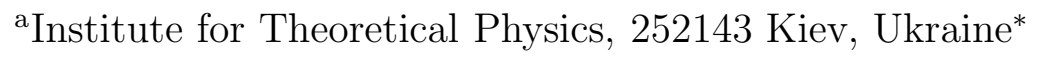 \\ ${ }^{\mathrm{b}}$ Chair of Quantum Field Theory, Physics Department, \\ Taras Shevchenko Kiev University, 252127 Kiev, Ukraine*
}

We study the possibility of existence of bound states for two interacting ${ }^{4} \mathrm{He}$ atoms. It is shown that for some potentials, there exist not only discrete levels but also bands akin to those in the Kronig-Penney model.

\section{INTRODUCTION}

It is known that most of the paradoxes of modern microscopic theory of superfluidity [1] have to do with the commonly accepted assumption of the single-particle Bose condensate (SBC) prevailing in the quantum structure of the superfluid component. In the ${ }^{4} \mathrm{He}$ Bose liquid, because of strong interaction, the SBC is strongly suppressed (it comprises no more than $10 \%$ of all ${ }^{4} \mathrm{He}$ atoms [2]), consequently it cannot be the basis of the superfluid component. If in some domain of momentum space there is strong enough attraction between atoms, bound pairs of bosons can form, whence a pair coherent condensate appears, and it is this condensate that will be the basis of the superfluid component.

The hypothesis of coupling of helium atoms below the $\lambda$ point may resolve some of those paradoxes $[1,3]$.

The main purpose of this paper is to explore the possibility of existence of bound states for two interacting ${ }^{4} \mathrm{He}$ atoms for different theoretical and phenomenological interatomic potentials. We will show that for some of the potentials, discrete levels do exist. Besides, we will analyze certain "quasi-crystallic" models, motivated by the conjecture that at temperatures below the $\lambda$ point, helium possesses a structure close to that of quantum crystals. Specifically, we will consider a model of atoms "smeared" over a sphere and a Kronig-Penney-type model. These models also allow for pairing of helium atoms; moreover, the Kronig-Penney-type model leads to a band of allowed energies.

\section{TWO INTERACTING ATOMS}

During several decades, different phenomenological potentials of interatomic interaction in gaseous and liquid ${ }^{4} \mathrm{He}$ have been constructed, based on empirical data on thermodynamic, kinetic and quantum mechanical properties of helium [4-8].

Consider the Schrödinger equation for two helium atoms ( $\Psi$ being the radial part of

*Email: mash@mashke.org; sivil@ap3.bitp.kiev.ua 
the wave function, $L=0$ )

$$
-\frac{\hbar^{2}}{2 m} \frac{1}{r^{2}} \frac{\partial}{\partial r}\left(r^{2} \frac{\partial}{\partial r} \Psi\right)+\Phi(r) \Psi=E \Psi,
$$

$\Phi(r)$ being the potential and $m$ the reduced mass $\left(2 m=m_{\mathrm{He}}=6.6466 \cdot 10^{-24} \mathrm{~g}\right)$. For a given $\Phi(r)$, we analyze Eq. (11) for discrete levels by means of solving it by the RungeKutta method from the left and from the right and trying to choose $\varepsilon$ such that the logarithmic derivatives of the two solutions match at some midpoint. If this fails, it is concluded that there is no level.

Table 1

Energy levels for two helium atoms assuming various interaction potentials

\begin{tabular}{|l|l|l|}
\hline Potential & $\Phi(r), 10^{-12} \operatorname{erg}(\mathrm{r}$ in $\AA)$ & Level, $\mathrm{K}$ \\
\hline \hline Rosen-Margenau [5] & $925 \exp (-4.4 r)-560 \exp (-5.33 r)-\frac{1.39}{r^{6}}-\frac{3}{r^{8}}$ & None \\
\hline Slatter-Kirkwood [6] & $770 \exp (-4.6 r)-\frac{1.49}{r^{6}}$ & None \\
\hline Intem-Schneider [8] & $1200 \exp (-4.72 r)-\frac{1.24}{r^{6}}-\frac{1.89}{r^{8}}$ & None \\
\hline Lennard-Jones & $\begin{array}{l}4 \epsilon\left[\left(\frac{\sigma}{r}\right)^{12}-\left(\frac{\sigma}{r}\right)^{6}\right] \\
\text { Case } 1[7]: \sigma=2.556 \AA, \epsilon=10.22 \mathrm{~K} \\
\text { Case } 2[9]: \sigma=2.642 \AA, \epsilon=10.80 \mathrm{~K}\end{array}$ & $\begin{array}{l}\text { None } \\
\text { Buckingham [10] }\end{array}$ \\
$\begin{array}{l}\left\{770 \exp (-4.6 r)-\frac{1.49}{r^{6}}, \quad r \leq 2.61 ;\right. \\
977 \exp (-4.6 r)-\frac{1.50}{r^{6}}-\frac{2.51}{r^{8}}, \quad r \geq 2.61\end{array}$ & 0.00632 \\
\hline Massey-Buckingham [10] & $1000 \exp (-4.6 r)-\frac{1.91}{r^{6}}$ & 0.0622 \\
\hline Buckingham-Hamilton [10] & $977 \exp (-4.6 r)-\frac{1.5}{r^{6}}-\frac{2.51}{r^{8}}$ & 0.00229 \\
\hline
\end{tabular}

\section{AN ATOM AT THE CENTER OF A LATTICE}

At temperatures below the $\lambda$ point, the structure and dynamical properties of helium are close to those of quantum crystals (e.g., Putterman [11] pointed out a possibility of transverse oscillations in superfluid helium). Motivated by this, let us study the behavior of a helium atom in the center of a lattice. For qualitative purposes, we replace the actual cubic lattice, with the lattice constant $d=4.5 \AA$, by $c=8$ atoms "smeared" over a sphere of radius $a=d \sqrt{3} / 2$. The potential corresponding to such a model is

$U(r)=\frac{c}{2} \int_{0}^{\pi} \Phi\left(\sqrt{r^{2}+a^{2}-2 r a \cos \theta}\right) \sin \theta d \theta$, 
where $\Phi$ is taken to be the Lennard-Jones potential (case 2 above). We then have to find a level in the potential $U(r)$, depicted below.

Obviously, since $U(r)$ is infinite at $r=a$, there will be infinitely many levels. We find the ground level to be $E=-11.45 \mathrm{~K}$, and the (certainly unphysical) first excited level to be $E=+35.64 \mathrm{~K}$.

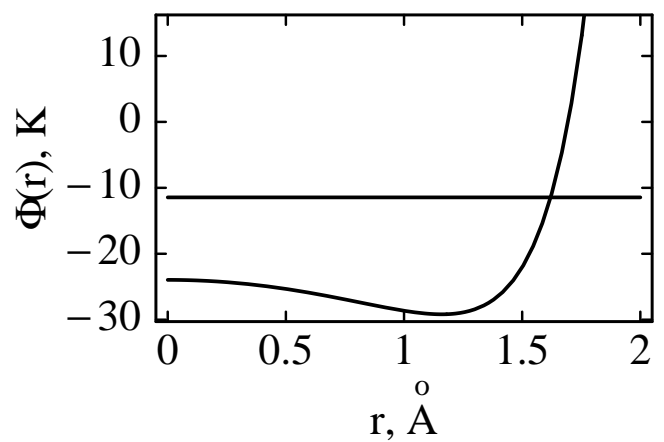

Fig. 1. The ground level for the atom in the center of a lattice.

\section{TWO NEIGHBORING CELLS AND A BAND}

Now, consider a "quasi-crystallic" model. A more realistic potential, without an infinite barrier, is

$\Phi_{2}(r)= \begin{cases}\Phi(r), & 0<r \leq a-d, \\ \Phi(a-d), & a-d<r \leq a+d, \\ \Phi(2 a-r), & a+d<r \leq 2 a .\end{cases}$

The parameter $d$ is taken to be $2.2 \AA$, which corresponds to twice the quantum chemical radius of the helium atom.

We get two levels: an even one with $E=-13.862 \mathrm{~K}$ and an odd one with $E=$ $-13.8486 \mathrm{~K}$.

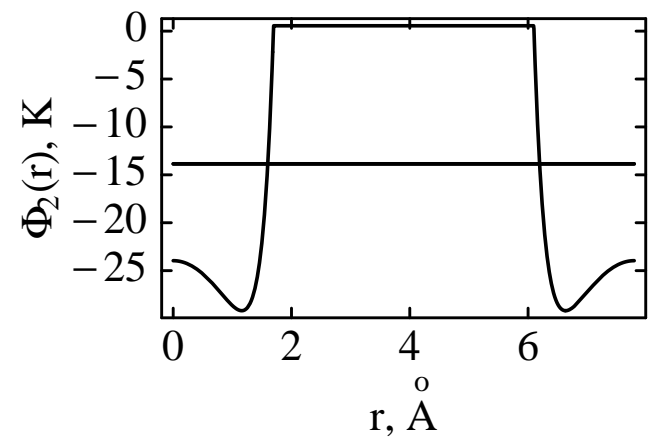

Fig. 2. The potential and the levels (the splitting is not noticeable) in the model of two neighboring cells.

Finally, consider a periodic potential:

$\Phi_{P}(r)=\Phi_{2}(r), \quad 0<r \leq 2 a ; \quad \Phi_{P}(r+2 a)=\Phi_{P}(r)$. 
We find the allowed band by a well-known method [12]: The wave function is represented as

$\psi(r)=\mathrm{e}^{\mathrm{i} K r} u_{K}(r)$,

where $u_{K}(r)$ is a periodic function. Matching the logarithmic derivative leads to

$\cos 2 K a=\frac{\left[u_{1}(0) u_{2}^{\prime}(a)+u_{1}(a) u_{2}^{\prime}(0)\right]-\left[u_{2}(0) u_{1}^{\prime}(a)+u_{2}(a) u_{1}^{\prime}(0)\right]}{2\left[u_{1}(0) u_{2}^{\prime}(0)-u_{2}(0) u_{1}^{\prime}(0)\right]}$,

where $u_{1}(r)$ and $u_{2}(r)$ are two arbitrary linearly independent solutions of the Schrödinger equation with energy $E$. The values of $E$ for which this equation can be solved for $K$ (that is, for which the right-hand side is not greater than 1 by absolute value), form an allowed band. Solving the Schrödinger equation numerically and plotting the $E$ dependence of the right-hand side, one finds the band and its boundaries at different values of $d$. The maximal width of the band, at $d \simeq 2.34$, is about $1 \mathrm{~K}$. Depicted below is the potential and the band at $d=2.33$.

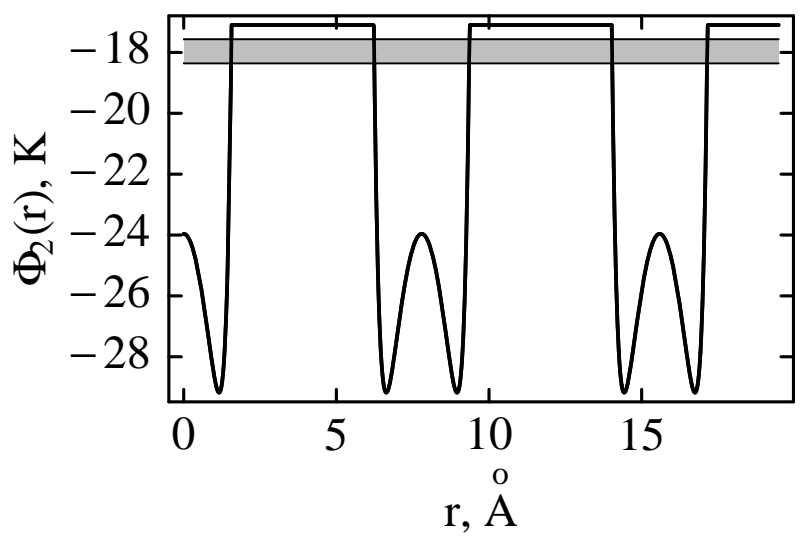

Fig. 3. The allowed band in the periodic potential model.

When $d$ is further increased, the upper edge of the band will "overflow" the potential and merge into the continuous spectrum. In fact, the band edges coincide with the even and odd levels in the two-well problem considered above. The reason is that the even and odd (with respect to $a$ ) wave functions of that problem (the boundary conditions for which are $\psi(0)=\psi(2 a)=0)$ can be extended into the antiperiodic $(\cos 2 K a=-1)$ and periodic $(\cos 2 K a=1)$ wave functions, respectively, of the periodic potential. According to the above, these functions correspond to the band edges.

For a rectangular potential (Kronig-Penney model [13]) of wells of width $a$ divided by barriers of height $V_{0}$ and width $b$ with the lattice constant being fixed: $a+b=4.5 \AA$, the band is determined by the equation

$\frac{\beta^{2}-\alpha^{2}}{2 \alpha \beta} \sinh \beta b \sin \alpha a+\cosh \beta b \cos \alpha a=\cos k(a+b)$,

where $\alpha=\sqrt{2 m E} / \hbar, \beta=\sqrt{2 m\left(V_{0}-E\right)} \hbar$. The values of $E$ for which the right-hand side is between -1 and 1 , form the allowed band. 
Table 2

Results $\left(E_{\text {low }}, E_{\text {high }}\right.$ and $\Delta E$ - lower edge, upper edge, and band width, respectively, all energies are in $\mathrm{K})$ :

\begin{tabular}{|c|c|c|c|c|}
\hline$a$ & $V_{0}$ & $E_{\text {low }}$ & $E_{\text {high }}$ & $\Delta E$ \\
\hline 2.5 & 10 & 2.15 & 2.35 & 0.20 \\
3.5 & 10 & 1.17 & 1.62 & 0.45 \\
3.5 & 5 & 0.78 & 1.56 & 0.78 \\
3.5 & 2 & 0.38 & 1.52 & 1.14 \\
4.0 & 2 & 0.20 & 1.92 & 1.72 \\
\hline
\end{tabular}

\section{CONCLUSION}

Thus, we have shown that in a superfluid Bose liquid, formation of bound pairs of bosons is possible — for some potentials, there are discrete levels. Moreover, it has been shown that bound states can also exist in more realistic models - the one of atoms "smeared" over the sphere and the periodic Kronig-Penney type model. Note in particular that the Kronig-Penney type model allows for the existence of bands. The presence of such bands may explain the observable critical velocities and the energy gap for the excitation spectrum curve ("S curve") in superfluid helium, which will be discussed in subsequent papers.

We are grateful to P.I. Fomin for suggesting the problem and constant encouragement and to A. Kostyuk for an interesting discussion and advice. Calculations have been made using Mathematica.

\section{REFERENCES}

1. E.A. Pashytskiy, Fiz. Nizk. Temp., 25 (1999) 115.

2. H.W.Jackson, Phys. Rev. A10 (1974) 278.

3. S.I. Vilchinskii, P.I. Fomin, Sov. J. Low Temp. Phys., 21 (1995) 7.

4. E.A. Mason, Journ. Chem. Phys., 22 (1954) 1678.

5. P. Rosen, Journ. Chem. Phys., 18 (1950) 1182; H. Margenau, Phys. Rev., 56 (1939) 1000; C.H. Page, Phys. Rev., 53 (1938) 426.

6. J.C. Slatter, J.G. Kirkwood, Phys. Rev., 37 (1931) 682.

7. J.O. Hirschfelder, Ch.F. Curtiss, R.B. Bird, Molecular Theory of Gases and Liquids, New York, 1954.

8. I.G. Kaplan, Introduction to the Theory of Intermolecular Interaction, Nauka, Moscow, 1982.

9. B. Plushkin (ed.), Molecular interaction from the diatomic molecules to the polymer, Mir, Moscow, 1981.

10. R.A. Buckingham, J. Hamilton, H.S.W. Massey, Proc. Roy. Soc., A179 (1941) 103.

11. S. Putterman, Superfluid Hydrodynamics, American Elsevier Publishing Company, Inc, New York, 1974.

12. S. Fluegge, Problems on Quantum Mechanics, Mir, Moscow, 1974, vol. 1., p. 75.

13. R. Kronig, W. Penney, Proc. Roy. Soc., 130 (1931) 499. 